\title{
A New Pyrazolate Osmium(VI) Nitrido Complex Exhibits Anticancer Activity Through Modulating Protein Homeostasis
}

Wen-Xiu Ni ( $\square$ wxni@stu.edu.cn )

Shantou University Medical College

Chengyang Huang

Shantou University Medical College

Wan-Qiong Huang

Shantou University Medical College

Peng-Chao Ji

Shantou University Medical College

Fu-Ling Song

Shantou University Medical College

Chuan-Xian Wang

Shantou University Medical College

Tao Liu

Shantou University Medical College

Meiyang Li

Shantou University Medical College

Hongzhi Guo

Shantou University Medical College

Cuicui Yu

Shantou University Medical College

Yong-Liang Huang

Shantou University Medical College

\section{Research Article}

Keywords: DNA, anticancer, cell, osmium

Posted Date: December 2nd, 2020

DOl: https://doi.org/10.21203/rs.3.rs-110247/v1 
License: (c) (i) This work is licensed under a Creative Commons Attribution 4.0 International License. Read Full License 


\title{
A new pyrazolate osmium(VI) nitrido complex exhibits anticancer activity through modulating protein homeostasis
}

\author{
Wan-Qiong Huang ${ }^{1,+}$, Peng-Chao $\mathrm{Ji}^{1,+}$, Fu-Ling Song ${ }^{1}$, Meiyang $\mathrm{Li}^{2}$, Hongzhi Guo ${ }^{2}$, Yong- \\ Liang Huang ${ }^{1}$, Cuicui $\mathrm{Yu}^{2}$, Chuan-Xian Wang ${ }^{1}$, Tao Liu ${ }^{1}$, Chengyang Huang ${ }^{2,4^{*}}$ and Wen- \\ Xiu $\mathrm{Ni}^{1,3^{*}}$
}

\author{
${ }^{1}$ Shantou University Medical College, Department of Medicinal Chemistry, Shantou, Guangdong, 515041, P. R. \\ China \\ ${ }^{2}$ Shantou University Medical College, Department of Physiology, Shantou, Guangdong, 515041, P. R. China \\ ${ }^{3}$ The First Affiliated Hospital of Shantou University Medical College, Clinical Research Centre, Shantou, \\ Guangdong, 515041, P. R. China \\ ${ }^{4}$ University of California, Eli and Edythe Broad Center for Regenerative Medicine and Stem Cell Research, David \\ Geffen School of Medicine Department of Biological Chemistry, Los Angeles California (UCLA) 90095, USA \\ *E-mail: wxni@stu.edu.cn, ORCID: 0000-0002-2370-6300 \\ *E-mail: cyhuang@stu.edu.cn \\ these authors contributed equally to this work
}

\begin{abstract}
Interest in the third-row transition metal osmium and its compounds as potential anticancer agents has grown in recent years. Here, we synthesized the osmium(VI) nitrido complex $\mathrm{Na}\left[\mathrm{Os}^{\mathrm{VI}}(\mathrm{N})(\mathrm{tpm})_{2}\right]$ (tpm= [5-(Thien-2-yl)-1H-pyrazol-3-yl]methanol) that does not have direct binding affinity to DNA. Cellular assays revealed that this new Os complex exhibited anticancer activity against cancer cell lines, cancer stem cells, and cisplatin-resistant cells. The Os complex $\mathrm{Na}\left[\mathrm{Os} \mathrm{sl}^{\mathrm{V}}(\mathrm{N})(\mathrm{tpm})_{2}\right] \mathrm{modulates}$ the expression of protein transportation-associated, DNA metabolism-associated and oxidative stress-associated proteins in HepG2 cells. Perturbation of protein expression activity by the Os complex in cancer cells affects the functions of the mitochondria and endoplasmic reticulum, resulting in high levels of cellular oxidative stress and low rates of cell survival. Moreover, it induced caspase-mediated apoptosis of HepG2 cancer cells. The study reveals a new high-valent Os complex as an anticancer agent candidate targeting cancer cell protein homeostasis.
\end{abstract}

\section{Introduction}

Cancer is a leading cause of death worldwide, ${ }^{1}$ and chemotherapy remains the outstanding effective strategy for prolonging patient survival. The clinical success of platinum-based anticancer drugs such as cisplatin, carboplatin, and oxaliplatin has stimulated extensive investigations into new metallodrugs with improved pharmacological properties, which may reduce side effects, such as kidney toxicity and nausea, and overcome drug resistance. ${ }^{2,3}$ Recently, other metal-based compounds with potential anti-cancer properties have been reported.$^{4,5}$ Ruthenium compounds are a potential alternative to platinum-based drugs due to their chemical and pharmacological properties. A number of ruthenium-based complexes have been reported to have promising anticancer activity, four of which have entered clinical trials. ${ }^{6,7}$ Osmium offers several features that are distinct from ruthenium, including a preference for higher stable oxidation states, stronger $\mathrm{p}$ back-donation from lower oxidation states, stronger spin-orbit coupling, and slower ligand exchange kinetics. In addition, osmium is relatively inert and stable under physiological conditions, making it a promising anticancer agent candidate. Sadler et al. reported a library of half-sandwich "pianostool" osmium(II) arene complexes that displayed increased anticancer activity. ${ }^{8}$ Our previous data showed that osmium(VI) nitrido compounds with tridentate Schiff bases ${ }^{9}$ and monodentate azole heterocycle ligands had anticancer properties in vitro and in vivo by targeting DNA strands. ${ }^{10}$ Lippard et al found that the cellular response evoked by antiproliferating osmium(VI) nitrido compounds with bidentate lipophilic N,N-chelating ligands could be tuned by subtle ligand modifications. ${ }^{11,12}$

Herein, we synthesized and characterized an osmium(VI) nitrido complex $\mathrm{Na}\left[\mathrm{Os}{ }^{\mathrm{VI}}(\mathrm{N})(\mathrm{tpm})_{2}\right]$ (tpm= [5-(Thien-2-yl)-1Hpyrazol-3-yl]methanol). We investigated its anticancer activity in eight human cell lines, including one cancer stem cell line, one cisplatin-resistant cell line and one normal liver cell line. The complex activated the caspase-mediated apoptosis pathway of HepG2 cells. Proteomic analysis suggested that HepG2 cells treatment with the Os complex regulated protein homeostasis, decreased the proteomic pathway of DNA repair and increased the pathways of DNA damage and DNA replication pressure, resulting in oxidative stress that was evidenced by an increase in reactive oxygen species (ROS), a reduction in mitochondrial membrane potential (MMP), and the induction of endoplasmic reticulum (ER) stress. Furthermore, the anticancer activity of the Os complex was examined in the nude mice model of cancer treatment, showing that the tumour size significantly decreased 


\section{Results}

\section{Synthesis and characterization of the Os complex.}

The reaction of $\left({ }^{n} \mathrm{Bu}_{4} \mathrm{~N}\right)\left[\mathrm{Os}^{\mathrm{VI}}(\mathrm{N}) \mathrm{Cl}_{4}\right]^{13}$ with two equiv. of [5-(Thien-2-yl)-1H-pyrazol-3-yl]methanol (tpm) in methanol took place in the presence of $\mathrm{NaOH}$. The final product $\mathrm{Na}\left[\mathrm{Os}^{\mathrm{VI}}(\mathrm{N})(\mathrm{tpm})_{2}\right]$ was characterized by elemental analysis, ${ }^{1} \mathrm{H} \mathrm{NMR}$ spectroscopy, infrared spectroscopy (IR) (Supplementary Fig. S1-2) and electrospray ionization mass spectrometry. Additionally, the X-ray crystal structure of $\mathrm{Na}\left[\mathrm{Os}^{\mathrm{VI}}(\mathrm{N})(\mathrm{tpm})_{2}\right](\mathbf{O s})$ was determined (Fig. 1 and Supplementary Tables S1-2). It showed that the osmium centre is coordinated by two deprotonated tpm ligands and one nitrido ligand. The Os $\equiv \mathrm{N}$ bond distance is 1.649(9) $\AA$ and the IR stretch for Os $\equiv \mathrm{N}$ occurs as a medium band at $1107 \mathrm{~cm}^{-1}$, which are comparable to other osmium nitrido complexes. ${ }^{14}$ The stability of the complex was observed by UV-visible spectroscopy, which showed that it was stable in DMSO for $72 \mathrm{~h}$ (Supplementary Fig. S3).

\section{Anticancer ability of the Os complex.}

The anticancer ability of the Os complex was investigated by screening its cytotoxicity in seven cancer cell lines, including HeLa (cervical), HepG2 (liver), A549 (lung), A2780 (ovarian), and MDA-MB-231 (breast) cells, a liver cancer stem cell line (HepG2-stem), and a cisplatin-resistant cell line (A549CIS). ${ }^{15,16}$ As shown in Table 1, the Os complex exhibited significant anti-proliferative activity with $\mathrm{IC}_{50}$ values in a range of $5.6-11.5 \mu \mathrm{M}$. The cytotoxicity of clinically used cisplatin after $48 \mathrm{~h}$ of treatment was also examined for comparison. We found that the Os complex robustly inhibited the growth of both the HepG2 and HepG2-stem cell lines, although it had a greater inhibitory effect on the cell viabilities of the cervical, ovarian, and breast cancer cell lines compared with cisplatin. We have also evaluated the cytotoxicity of Os towards human normal liver cell line (LO-2). It found that Os is less cytotoxic than cancer cells with $\mathrm{IC}_{50}$ value of $28.5 \mu \mathrm{M}$. Notably, the Os complex can also reduce the viability of cisplatin-resistant cells. This complex is nearly as effective in cisplatin-resistant cells with a resistance factor (RF) of 1.3. The RF determined for cisplatin was 12.2, which is 9.4-fold greater than Os, indicating that the Os complex may have a different anticancer mechanism than that of cisplatin. Cisplatin has been found to react with DNA strands. CisplatinDNA lesions can block the transcription of genes, resulting in cell apoptosis. ${ }^{17,18}$ Consistent with our hypothesis, we examined the interaction of Os with DNA by means of DNA migration but found no apparent effect on the electrophoretic mobility of plasmid DNA in agarose gel, suggesting that DNA was not the major target of Os (Supplementary Fig. S4).

\section{Proteomics analysis.}

To gain insight into the molecular effects of the Os complex on HepG2 cells, we performed proteomic analysis of cells after 8 $\mathrm{h}$ and $24 \mathrm{~h}$ of treatment with the Os complex by mass spectrometry. Gene ontology (GO) analysis revealed that the expression of proteins in the cells after $8 \mathrm{~h}$ and $24 \mathrm{~h}$ of treatment had distinct proteomic features (Fig. 2A). After $8 \mathrm{~h}$ of treatment with the Os complex, the expression levels of proteins involved in protein transportation and localization decreased by at least 1.5 -fold while proteins involved in cytoskeletal organization and morphogenesis of the epithelium increased by at least 1.5-fold, indicating that the Os complex altered the dynamics of the cells and affected cell infiltration. Interestingly, $24 \mathrm{~h}$ of treatment with the Os complex decreased the expression of proteins involved in DNA metabolism, the cell cycle, nucleic acid metabolism, and DNA damage repair while increasing protein expression for the oxoacid, amide, and antibiotic metabolic process and the response to unfolded proteins (Fig. 2A). The top categories from the GO analysis of up- and downregulated proteins after $24 \mathrm{~h}$ of treatment with the Os complex were oxoacid and DNA metabolic processes, respectively. All of the proteins in these two categories are shown in the heat map (Fig. 2B). Proteins involved in oxoacid metabolism play key roles in cancer treatment. ${ }^{19,20}$ The DNA metabolic process contains a large number of proteins associated with DNA replication and DNA damage, which may be the molecular mechanisms behind the observed increased cell apoptosis after drug treatment. ${ }^{21}$ In the case of the DNA metabolic process, the abundances of 27 proteins were significantly altered. These proteins play a role in DNA replication and repair (Fig. 2B). Reduced expression of these genes may lead to the accumulation of DNA damage to cells and reduce cell proliferation, which in turn promotes cell apoptosis. ${ }^{21}$

In the case of the oxoacid metabolic process, the expression of GCLM increased greatly (Fig. 2B). The glutamate-cysteine ligase regulatory subunit is encoded by the GCLM gene and is the first-rate limiting enzyme in glutathione synthesis. In response to various oxidative stresses, GCLM can be regulated through the electrophile response element (EPRE). ${ }^{22}$ EPRE has been found to be an indicator of oxidative stress. ${ }^{23}$ Our data suggest that the Os complex regulates the cellular redox state to affect the survival of cancer cells. Moreover, DDB2 expression greatly decreases after Os treatment. DDB2 interacts with DDB1 to form a UV-DDB complex that senses UV-induced DNA damage and initiates DNA repair through nucleotide resection (the NER pathway) and repair. ${ }^{24}$ Interestingly, DDB2 can inhibit the proliferation and migration of cancer cells mediated through PAQR3. ${ }^{25}$ In addition, DDB2 directly interacts with LRH-1 and facilitates LRH-1 protein ubiquitination and degradation, which are involved in sugar and lipid metabolism and protein ubiquitination. ${ }^{26}$ The decreased expression of DDB2 after Os treatment suggests that these DDB2 pathways may be impaired, resulting in cellular damage. Another protein that is highly upregulated after Os complex treatment is KYNU. The kynurenine pathway is the major metabolic pathway of the amino acid tryptophan. Kynureninase or L-kynurenine hydrolase (KYNU) is an important enzyme in this pathway. Overall, these data consistently suggest that treatment of HepG2 cells with the Os complex decreases the DNA repair capacity while increasing DNA damage accumulation and DNA replication pressure, resulting in cellular oxidative stress and cell apoptosis. The DNA damage is also confirmed by Western blotting as shown in Fig. 3, the Os complex can activate the phosphorylated histone $\gamma$-H2AX, an early DNA damage response marker. 
Overall, these data consistently suggest that treatment of cancer cells with the Os complex decreases the DNA repair capacity while increasing DNA damage accumulation and DNA replication pressure, resulting in cellular oxidative stress and cell apoptosis.

\section{Effects of the Os complex on apoptosis.}

In order to investigate whether the Os complex triggers cell apoptosis, HepG2 cancer cells and HepG2-stem cancer stem cells were treated with the Os complex at 4,8 , and $12 \mu \mathrm{M}$ for $24 \mathrm{~h}$ and subjected to flow cytometry analysis. As shown in Supplementary Fig. S5, in an Os complex concentration-dependent manner, the apoptotic (early and late apoptotic cells) proportion of HepG2 cancer cells increased from $5.21 \%$ to $38.35 \%$, where late apoptosis was more significant. Compared with the control treated with DMSO, apoptosis of HepG2-stem cells treated with the Os complex significantly increased from 5.02\% to $64.9 \%$, which was attributed equally to both early and late apoptosis (Supplementary Fig. S5). These results suggested that cell death after treatment with the Os complex was mainly induced through apoptosis. Caspases-8/9 are the initiator caspases of extrinsic apoptosis, while caspase-3 is an executioner of apoptosis. In addition, cleavage of poly-(ADP-ribose)-polymerase1 (PARP-1) is one of the imperative indicators for caspase-mediated apoptosis. To investigate whether Os-induced apoptosis is mediated by caspases, we examined the protein levels of caspases and PARP-1 in Os-treated HepG2 cells by Western blotting. As shown in Fig. 3, lower concentrations of Os displayed the sequential activation of caspases 8, 9, and 3, which indicated that the Os complex can induce cell apoptosis involving extrinsic (death ligand) and intrinsic (mitochondrial) pathways. ${ }^{27}$ From the perspective of cell morphology, as shown in Supplementary Fig. S6, the cells were observed to gradually shrink, become round and fall off, which is consistent with the morphological characteristics of apoptosis.

\section{Mitochondrial membrane potential (MMP) and endoplasmic reticulum (ER) stress.}

Evidence has confirmed that the Os complex induces apoptosis via a caspase-3/9-dependent pathway. To further investigate the upstream signaling pathways in HepG2 cells, the cells were exposed to the Os complex to detect the MMP and levels of ROS production. The red/green fluorescence of 5,5',6,6'-tetrachloro-1,1'-3,3'-tetraethyl-benzimidazolylcarbocyanine iodide (JC-1), a mitochondria-selective fluorescent probe, was detected by confocal microscopy (Supplementary Fig. S7) and flow cytometry (Supplementary Fig. S8). When the mitochondrial membrane potential is high, JC-1 accumulates in the matrix of the mitochondria to form polymers (J-aggregates) with red fluorescence. When the mitochondrial membrane potential is low, JC-1 exists as a monomer with a green fluorescence signal. As shown in Supplementary Fig. S7, significant red fluorescence was observed in HepG2 cells in the control group, and only green fluorescence was detected in the positive control group treated with carbonyl cyanide m-chlorophenylhydrazone (CCCP). Increasing concentrations of the Os complex, the red fluorescence decreased and the green fluorescence increased. When up to $13 \mu \mathrm{M}$, the $\mathbf{O s}$ complex caused significant upregulation of intensity in monomer. The decline in mitochondrial membrane potential (MMP) indicated that the early stages of apoptosis were induced by the Os complex. Reactive oxygen species (ROS) are the main molecules produced by oxidative stress in the body and have been recognized as important factors in tumorigenesis, tumor development and tumor recurrence. In recent years, studies have found that ROS and cell apoptosis are closely related. ${ }^{28}$ Therefore, progressive concentrations of the Os complex were added to HepG2 cells for $3 \mathrm{~h}$, and an inverted fluorescence microscope was used to detect the oxidation of the sensitive fluorescence probe DCFH-DA by measuring the fluorescence intensity to test the content of intracellular ROS. As shown in Supplementary Fig. S9, compared with the negative control group, the fluorescence intensity of each treatment group obviously increased, which indicated that the complex promoted HepG2 cells to release ROS, thus inducing cell apoptosis and suppressing proliferation. We further determined whether the Os complex induced endoplasmic reticulum (ER) stress. Glucose-regulated protein 78 (GRP78/Bip) is induced during ER stress, which is involved in polypeptide translocation across the ER membrane, and acts as an apoptotic regulator by protecting the host cell against ER stress-induced cell death. A high level of GRP78 protein expression is indicative of ER stress. ${ }^{29}$ At progressive concentrations of the Os complex (4, 8, and $\left.12 \mu \mathrm{M}\right)$, endoplasmic reticulum (ER) stress was activated, as evidenced by the increased expression level of the GRP78 protein. (Fig. 3)

\section{In vivo antitumour activity.}

To reveal the anticancer activity of the Os complex in vivo, we examined the therapeutic effects of Os in a human cancer xenografted in nude mouse model. We successfully established a xenograft tumour model in nude mice using HepG2 cells. HepG2 cell-bearing nude mice were randomly divided into the vehicle control group, two Os-treated groups $(0.2 \mathrm{and} 1 \mathrm{mg} / \mathrm{kg})$ and one cisplatin-treated group $(1 \mathrm{mg} / \mathrm{kg})$. Mice were treated with the different complexes via intravenous tail injection every 4 days. The tumour volumes and the mouse weights were measured every 2 days. After 24 days of treatment, the tumours from each group were excised and weighed. As shown in Fig. 4A-C, the mice treated with the Os complex displayed a significant reduction in both tumour volume and weight compared to the vehicle control group. Dose-dependent inhibition of tumour growth after Os treatment at 0.2 and $1 \mathrm{mg} / \mathrm{kg}$ showed relative tumour regression rates of $30.6 \%$ and $55.8 \%$, respectively. The inhibitory effect of Os on tumour growth was similar to that of cisplatin (57.8\%) at the same concentration. Moreover, the body weights of the mice treated with Os did not decrease significantly (Fig. 4), indicating that this complex possesses low toxicity compared with cisplatin. Together, our results suggest that the Os complex retains anticancer activity in vivo.

\section{Conclusion}

In summary, an osmium(VI) nitrido complex bearing a pyrazolate ligand was synthesized and characterized. The Os complex exhibits anticancer activity against cancer cell lines, cancer stem cells, and cisplatin-resistant cells by inducing cell apoptosis. In addition, caspase activation and oxidative stress in the cells after Os treatment suggests that apoptosis occurs through extrinsic (death ligand) and intrinsic (mitochondrial) pathways. Although the Os complex does not directly target DNA, it can regulate protein homeostasis by mobilizing different functional branches of the protein network at different time points during treatment. 
Protein transportation-associated proteins were significantly downregulated at earlier time points after Os treatment. Furthermore, DNA metabolism-associated and oxidative stress-associated proteins were significantly downregulated and upregulated, respectively, at later time points after Os treatment, suggesting that the Os complex can decrease DNA repair capacity while increasing DNA damage accumulation and DNA replication pressure, which may increase oxidative stress in the cells. Our study reveals a new high-valent Os complex as a promising anticancer agent candidate targeting protein homeostasis.

\section{Methods}

Instrumentation. UV-visible spectra were recorded on a Shimadzu UV2450-2550 spectrophotometer in $1 \mathrm{~cm}$ cuvettes. Infrared spectrum was obtained from KBr plates using a Nicolet AVATAR 360 FTIR spectrophotometer. ${ }^{1} \mathrm{H}-\mathrm{NMR}$ spectrum was recorded on a Bruker DPX 400 spectrometer (Bruker, Karlsruhe, Germany). Elemental analysis was done on a Vario EL cube CHNs analyzer (Elementar, Germany). X-ray Crystallography was used a SMART CCD (Bruker, Germany).

Materials. 3-(4,5-Dimethyl-2-thiazolyl)-2,5-diphenyl-2H-tetrazolium bromide (MTT) and Cisplatin purchased from Alfa and used as received. pBR322 DNA was purchased from Thermo Scientific. FITC Annexin V Appoptosis Detection Kit I was purchased from BD Pharmingen ${ }^{\mathrm{TM}}$. Reactive Oxygen Species Assay Kit and Mitochondrial membrane potential assay kit with JC-1 was obtained from Beyotime. $\beta$-Actin, Phospho-Histone H2A.X, Caspase 3, PARP1(CST, rabbit, dilution 1:1000), caspase 8(Abcam, rabbit, dilution 1:1000), caspase 9(Abcam, rabbit, dilution 1:2000), GRP78(SANTA CRUZ BIOTECHNOLOGY) were used as primary antibodies and HRP AffiniPure Goat Anti-Rabbit (BOSTER, dilution 1:5000) was used as secondary antibody.

Synthesis of Complex. $\left[{ }^{\mathrm{n}} \mathrm{Bu}_{4} \mathrm{~N}\right]\left[\mathrm{Os}^{\mathrm{VI}}(\mathrm{N})(\mathrm{Cl})_{4}\right](235.2 \mathrm{mg}, 0.4 \mathrm{mmol})$ and [5-(Thien-2-yl)-1H-pyrazol-3-yl]methanol) (144.2 $\mathrm{mg}, 0.8 \mathrm{mmol}$ ) were fixed and added $15 \mathrm{ml}$ of methanol at room temperature, stirring, after being their completely dissolved, with $5 \mathrm{mg} / \mathrm{ml}$ of $\mathrm{NaOH}$ of methanol solution to adjust the $\mathrm{pH}$ to 9 . The mixture was continued to stir at room temperature for $12 \mathrm{~h}$, the result yellow precipitate was filtered out, using methanol washing and vacuum drying. The filtrate was volatilized at room temperature. After a few weeks the needle yellow transparent crystal was obtained. Yield: $81.6 \%$. IR(KBr, v/cm $\left.{ }^{-1}\right): v$ $(\mathrm{Os} \equiv \mathrm{N}) 1107 \mathrm{~cm}^{-1} ; v(\mathrm{C}=\mathrm{N}) 146 \mathrm{~cm}^{-1} ; v(\mathrm{C}(=\mathrm{C})-\mathrm{H}) 3125 \mathrm{~cm}^{-1} .{ }^{1} \mathrm{H}$ NMR $\left(400 \mathrm{MHz}, \mathrm{d}_{6}-\mathrm{DMSO}\right): \delta 7.46(\mathrm{~s}, 1 \mathrm{H}), \delta 7.35(\mathrm{~s}, 1 \mathrm{H}), \delta$ $7.07(\mathrm{~m}, 1 \mathrm{H}), \delta 5.2(\mathrm{~d}, 1 \mathrm{H}), \delta 4.9(\mathrm{~d}, 1 \mathrm{H})$. $\mathrm{CHN}$, found: $\mathrm{C}, 33.04 ; \mathrm{H}, 2.50 ; \mathrm{N}, 11.41 ; \mathrm{S}, 10.48$. Calcd. for $\mathrm{C}_{16} \mathrm{H}_{12} \mathrm{~N}_{5} \mathrm{NaO}_{2} \mathrm{~S}_{2} \mathrm{Os} \cdot \mathrm{CH}_{3} \mathrm{OH}: \mathrm{C}, 33.16 ; \mathrm{H}, 2.62 ; \mathrm{N}, 11.37 ; \mathrm{S}, 10.42$.

X-ray crystallography. Suitable single crystals were mounted with glue at the end of a glass fiber. X-ray diffraction data were collected on a XtaLab PRO MM007HF DW Diffractometer System equipped with a MicroMax-007DW Micro Focus X-ray generator and Pilatus 200K silicon diarray detector (Rigaku, Japan, $\mathrm{Cu} \mathrm{K} \alpha, \lambda=1.54184 \AA$ ) under $293 \mathrm{~K}$. Data reductions were performed on CrysAlisPro 1.171.39.28b (Rigaku OD, 2015). Structure solution was carried out using SHELXT and refinement with SHELXL, within the OLEX2 graphical interface. ${ }^{30-32}$ Restraints (SADI and DFIX) were applied for disordered methanol molecule. All non-hydrogen atoms were refined first isotropically and then anisotropically. All of the hydrogen atoms of the ligands were placed in calculated positions with fixed isotropic thermal parameters and included in the structure factor calculations in the final stage of full-matrix least-squares refinement. CCDC no. 2009053 contains the supplementary crystallographic data for the complex $\mathrm{Na}\left[\mathrm{Os}{ }^{\mathrm{VI}}(\mathrm{N})(\mathrm{tpm})_{2}\right] \cdot \mathrm{CH}_{3} \mathrm{OH}$. All data can be obtained free from The Cambridge Crystallographic Data Centre.

Cell culture conditions. ${ }^{33}$ Cell lines showed in this work including liver hepatocellular carcinoma (HepG2) and its stem cell (HepG2-stem), cervical epithelioid carcinoma (HeLa), lung carcinoma (A549) and its cisplatin-resistant daughter cell (A549CIS), breast adenocarcinoma (MDA-MB-231) and ovarian carcinoma (A2780). All cells were cultured at $37^{\circ} \mathrm{C} / 5 \% \mathrm{CO} 2$. A549, A549CIS, HeLa and MDA-MB-231 cells were grown in Dulbecco's Modified Eagle's Medium (DMEM; Gibco), HepG2 and A2780 cells were grown in Roswell Park Memorial Institute (RPMI 1640; Gibco). The medium was supplemented with $10 \%$ fetal bovine serum(Gibco), 1\% GlutaMAX (Gibco) and 1\% penicillin-streptomycin (Gibco). Human normal liver cell line LO-2 (HL-7702) was purchased from the Cell Bank of the Chinese Academy of Sciences (Shanghai, China). LO-2 was grown in RPMI 1640, supplemented with $20 \%$ fetal bovine serum, $1 \%$ GlutaMAX, and $1 \%$ penicillin-streptomycin.

MTT assay. Cells were rinsed with PBS and digested with $0.25 \%$ trypsin-EDTA(Gibco). Then cells with the density of $5 \times 10^{4}$ cells / $\mathrm{ml}$ were counted and added into the 96-well plate. Cells were incubated for $24 \mathrm{~h}$, and then the solution containing the tested complex and positive control cisplatin were added. After $48 \mathrm{~h}$, drug solutions were replaced by $0.5 \mathrm{mg} / \mathrm{ml} \mathrm{MTT}$ solution. After incubation for $1.5 \mathrm{~h}$ at $37^{\circ} \mathrm{C}$, old solution was removed and DMSO was added, the 96-well plate was vortexed for 15 min in the dark. OD values at a wavelength of $570 \mathrm{~nm}$ were read by Infinite M200 (Swiss, Tecan). The curve is fitted using the logarithmic interpolation in Origin 8.0 software and the half inhibition concentration $\left(\mathrm{IC}_{50}\right)$ is calculated. Each experiment was repeated three times.

Proteomic analysis. HepG2 cells were treated with Os compound at $6.5 \mu \mathrm{M}$ concentration for 8 and $24 \mathrm{~h}$ in a $\mathrm{CO}_{2}$ incubator at $37{ }^{\circ} \mathrm{C}$. Equal amount of DMSO were added to HepG2 cells as a positive control. For each sample, three biological replicates were prepared. HPLC-MS/MS analysis were performed as described. ${ }^{34,35}$ The differentially expressed proteins (1.5-fold changes after the Os complex treatment) identified by mass spectrometry analysis are subjected to gene ontology (GO) analysis as described previously. ${ }^{36}$ The differentially expressed proteins (1.5-fold changes after the Os complex treatment) identified by mass spectrometry analysis are subjected to gene ontology (GO) analysis as described previously. ${ }^{36}$ The top 6 GO terms under 
the biological process category were retrieved for profiling the functional enrichment of up and down regulated gene products, respectively.

Western Blot Analysis. HepG2 cells were seeded into the six-well plate. After being cultured in the cell incubator for $24 \mathrm{~h}, 4$, 8 and $12 \mu \mathrm{M}$ of Os complex were prepared to treat the cells for $24 \mathrm{~h}$. The cells were collected, centrifuged, and washed with PBS twice, then cell lysis buffer with proteas inhibitor Phenylmethanesulfonyl fluoride(PMSF) was added to the cell precipitation and lysed for 15 minutes. Protein concentration was detected with BCA Protein Assay Kit (Beyotime), and the detection wavelength was set at $562 \mathrm{~nm}$ by Infinite M200 (Swiss, Tecan). The proteins were denaturted by boiling, and equal amount of proteins were calculated for protein electrophoresis, transferred to nitrocellulose membrane and then blocking. The membranes were cut according to the molecular weight of the proteins and incubated with primary antibody at $4{ }^{\circ} \mathrm{C}$ overnight. The secondary antibody was incubated for $1.5 \mathrm{~h}$. Next, the ECL detection kit (Beyotime) was used for exposure imaging on a High ChemiDoc XRS (Bio-Rad ChemiDoc XRS+, USA).

Xenograft in nude mice. Nude mice were purchased from the Hunan SJA Laboratory Animal Co. Ltd (Hunan, China). All nude mice assays were strictly obeyed the "Guidelines on the Treatment of Experimental Animals" issued by the Ministry of Science, the "Regulations on the Administration of Experimental Animals in Guangdong Province" issued by the Standing Committee of the National People's Congress of Guangdong Province (2010) 41, Rev. 2019, Technology of China (2006) 398 and was approved by the Institutional Animal Care and Use Committee of Shantou University Medical College (SUMC). The quality certificate is NO.43004700043812. The License number is SYXK (Guangdong) 2017-0079. HepG2 cells were subcutaneously injected into Nude mice (4 weeks old). Tumor size was surveyed with digital caliper and calculated with the formula, Tumor volume $\left(\mathrm{mm}^{3}\right)=$ longest diameter $(\mathrm{mm}) \times[\text { shortest diameter }(\mathrm{mm})]^{2} / 2$. After 5 days, HepG2-bearing nude mice were randomly classified as four treatment groups. Mice were administered with complex at the dose of $0.2 \mathrm{and} 1 \mathrm{mg} / \mathrm{kg}$ body weight and $1 \mathrm{mg} / \mathrm{kg}$ cisplatin via injection at tail intravenous every 4 days. The mice weight and the tumor volume were detected every 2 days. At the end of the studied period ( 29 days), the treated mice were scarified and separated the tumor nodules. The tumors were weighed and photographed. The tumor growth inhibition rate was calculated with the following formulas:

Tumor growth inhibition rate $(\%)=(1-$ mean tumor weight of the treatment / mean tumor weight of the negative group $) \times$ $100 \%$;

All datas were displayed in Mean \pm SD, using SPSS for statistical analysis.

\section{References}

1. Siegel, R. L., Miller, K. D. \& Jemal, A. Cancer Statistics, 2017. CA Cancer J. Clin. 67, 7-30, doi:10.3322/caac.21387 (2017).

2. Deo, K. M. et al. Platinum coordination compounds with potent anticancer activity. Coord. Chem. Rev. 375, 148-163, doi:10.1016/j.ccr.2017.11.014 (2018).

3. Meier-Menches, S. M., Gerner, C., Berger, W., Hartinger, C. G. \& Keppler, B. K. Structure-activity relationships for ruthenium and osmium anticancer agents - towards clinical development. Chem. Soc. Rev. 47, 909-928, doi: $10.1039 / \mathrm{c} 7 \operatorname{cs} 00332 \mathrm{c}(2018)$.

4. Luo, H. J., Cao, B., Chan, A. S. C., Sun, R. W. Y. \& Zou, T. T. Cyclometalated Gold(III)-Hydride Complexes Exhibit Visible Light-Induced Thiol Reactivity and Act as Potent Photo-Activated Anti-Cancer Agents. Angew. Chem., Int. Ed. 59, 11046-11052, doi:10.1002/anie.202000528 (2020).

5. Zhang, J.-L. et al. A Gallium(III) Complex that Engages Protein Disulfide Isomerase A3 (PDIA3) as an Anticancer Target. Angew. Chem., Int. Ed., doi:10.1002/anie.202008432 (2020).

6. Thota, S., Rodrigues, D. A., Crans, D. C. \& Barreiro, E. J. Ru(II) Compounds: Next-Generation Anticancer Metallotherapeutics? J. Med. Chem., doi:10.1021/acs.jmedchem.7b01689 (2018).

7. Zeng, L. L. et al. The development of anticancer ruthenium(II) complexes: from single molecule compounds to nanomaterials. Chem. Soc. Rev. 46, 5771-5804, doi:10.1039/c7cs00195a (2017).

8. Needham, R. J. et al. In-Cell Activation of Organo-Osmium(II) Anticancer Complexes. Angew. Chem., Int. Ed. 56, 10171020, doi:10.1002/anie.201610290 (2017).

9. Ni, W. X. et al. Osmium(VI) complexes as a new class of potential anti-cancer agents. Chem. Commun. 47, 2140-2142, doi:10.1039/c0cc04515b (2011).

10.Ni, W.-X. et al. Osmium(vi) nitrido complexes bearing azole heterocycles: a new class of antitumor agents. Chem. Sci. 3, 1582, doi:10.1039/c2sc01031c (2012).

11. Suntharalingam, K. et al. Bidentate ligands on osmium(VI) nitrido complexes control intracellular targeting and cell death pathways. J. Am. Chem. Soc. 135, 14060-14063, doi:10.1021/ja4075375 (2013).

12. Berger, G. et al. Anticancer activity of osmium(VI) nitrido complexes in patient-derived glioblastoma initiating cells and 
in vivo mouse models. Cancer Lett 416, 138-148, doi:10.1016/j.canlet.2017.11.041 (2018).

13.Leung, C.-F., Yiu, D. T. Y., Wong, W.-T., Peng, S.-M. \& Lau, T.-C. Synthesis and reactivity of osmium (VI) nitrido complexes containing pyridine-carboxylato ligands. Inorganica Chim. Acta. 362, 3576-3582, doi:10.1016/j.ica.2009.04.002 (2009).

14. Che, C.-M. \& Lau, T.-C. in Comprehensive Coordination Chemistry II (eds Jon A. McCleverty \& Thomas J. Meyer) 733847 (Pergamon, 2003).

15. Mosmann, T. Rapid colorimetric assay for cellular growth and survival: application to proliferation and cytotoxicity assays. J. Immunol. Methods 65, 55-63 (1983).

16.Liang, X. J. et al. Metallofullerene nanoparticles circumvent tumor resistance to cisplatin by reactivating endocytosis. Proc. Natl. Acad. Sci. U S A 107, 7449-7454, doi:10.1073/pnas.0909707107 (2010).

17.Johnstone, T. C., Suntharalingam, K. \& Lippard, S. J. The Next Generation of Platinum Drugs: Targeted Pt(II) Agents, Nanoparticle Delivery, and Pt(IV) Prodrugs. Chem. Rev. 116, 3436-3486, doi:10.1021/acs.chemrev.5b00597 (2016).

18.Johnstone, T. C., Suntharalingam, K. \& Lippard, S. J. Third row transition metals for the treatment of cancer. Philos. Trans. A, Math., Phys. Eng. Sci. 373, doi:10.1098/rsta.2014.0185 (2015).

19. Olson, K. A., Schell, J. C. \& Rutter, J. Pyruvate and Metabolic Flexibility: Illuminating a Path Toward Selective Cancer Therapies. Trends Biochem. Sci. 41, 219-230, doi:10.1016/j.tibs.2016.01.002 (2016).

20.Gray, L. R., Tompkins, S. C. \& Taylor, E. B. Regulation of pyruvate metabolism and human disease. Cell. Mol. Life Sci. CMLS 71, 2577-2604, doi:10.1007/s00018-013-1539-2 (2014).

21.Roos, W. P. \& Kaina, B. DNA damage-induced cell death by apoptosis. Trends Mol. Med. 12, 440-450, doi:10.1016/j.molmed.2006.07.007 (2006).

22. Moinova, H. R. \& Mulcahy, R. T. An electrophile responsive element (EpRE) regulates beta-naphthoflavone induction of the human gamma-glutamylcysteine synthetase regulatory subunit gene. Constitutive expression is mediated by an adjacent AP-1 site. J. Biol. Chem. 273, 14683-14689 (1998).

23.Zipper, L. M. \& Mulcahy, R. T. Erk activation is required for Nrf2 nuclear localization during pyrrolidine dithiocarbamate induction of glutamate cysteine ligase modulatory gene expression in HepG2 cells. Toxicol. Sci. 73, 124-134, doi:10.1093/toxsci/kfg083 (2003).

24.Tang, J. \& Chu, G. Xeroderma pigmentosum complementation group E and UV-damaged DNA-binding protein. DNA repair 1, 601-616 (2002).

25. Qiao, S. et al. DDB2 is involved in ubiquitination and degradation of PAQR3 and regulates tumorigenesis of gastric cancer cells. Biochem. J. 469, 469-480, doi:10.1042/bj20150253 (2015).

26.Lai, T. C. \& Hu, M. C. Regulation of liver receptor homologue-1 by DDB2 E3 ligase activity is critical for hepatic glucose metabolism. Sci. Rep. 9, doi:Artn 5304, 10.1038/S41598-019-41411-X (2019).

27. Shalini, S., Dorstyn, L., Dawar, S. \& Kumar, S. Old, new and emerging functions of caspases. Cell Death Differ. 22, 526539, doi:10.1038/cdd.2014.216 (2015).

28.Sinha, K., Das, J., Pal, P. B. \& Sil, P. C. Oxidative stress: the mitochondria-dependent and mitochondria-independent pathways of apoptosis. Arch. Toxicol. 87, 1157-1180, doi:10.1007/s00204-013-1034-4 (2013).

29. Cai, Y. et al. Betulinic acid chemosensitizes breast cancer by triggering ER stress-mediated apoptosis by directly targeting GRP78. Cell Death Dis. 9, 636, doi:10.1038/s41419-018-0669-8 (2018).

30. Sheldrick, G. M. SHELXT - integrated space-group and crystal-structure determination. Acta Cry. A Found Adv. 71, 3-8, doi:10.1107/s2053273314026370 (2015).

31. Sheldrick, G. M. Crystal structure refinement with SHELXL. Acta crystallographica. Section C, Structural chemistry 71, 3-8, doi:10.1107/s2053229614024218 (2015).

32.Dolomanov, O. V., Bourhis, L. J., Gildea, R. J., Howard, J. A. K. \& Puschmann, H. OLEX2: a complete structure solution, refinement and analysis program. Journal Appl. Crystallogr. 42, 339-341, doi:10.1107/s0021889808042726 (2009).

33.Huang W.Q., Wang C.X., Liu T., Li Z.X., Pan C., Chen Y.Z., Lian X., Man W.L. \& Ni W.X. A cytotoxic nitridoosmium(VI) complex induces caspase-mediated apoptosis in HepG2 cancer cells. Dalton transactions, doi:10.1039/d0dt02715d (2020).

34.Rappsilber, J., Mann, M. \& Ishihama, Y. Protocol for micro-purification, enrichment, pre-fractionation and storage of peptides for proteomics using StageTips. Nat. Protoc. 2, 1896-1906, doi:10.1038/nprot.2007.261 (2007).

35.Rappsilber, J., Ishihama, Y. \& Mann, M. Stop and go extraction tips for matrix-assisted laser desorption/ionization, nanoelectrospray, and LC/MS sample pretreatment in proteomics. Anal. Chem. 75, 663-670, doi:10.1021/ac026117i (2003). 
36. Huang, C. et al. Cbx3 maintains lineage specificity during neural differentiation. Genes Dev. 31, 241-246, doi:10.1101/gad.292169.116 (2017). 


\section{Acknowledgements (not compulsory)}

This study was supported by the Guangdong Major Project of Basic and Applied Basic Research (no. 2019B030302009), the National Natural Science Foundation of China (no. 81671396, 21401125), funding from the "Tezhi Project" (no.

201627025) and "Yangfan Project" (no. 201635031) of Guangdong Province, the Natural Science Foundation of Guangdong Province grant 2017A030313780, the Special Technological Fund of Guangdong Province (no. 190823175554386), and the Li Ka Shing Foundation Cross-Disciplinary Research Grant (2020LKSFG01F). We thank Guangdong Provincial Key Laboratory of Infectious Diseases and Molecular Immunopathology.

\section{Author contributions statement}

Dr. Wen-Xiu Ni and Dr. Chengyang Huang planned the project, supervised all experiments, and wrote the manuscript. Wenxiu-Ni also performed the sample preparation and mass spectrometry analysis for proteomics section. All experiments were performed, data were analyzed and interpreted by the other authors, detail information is shown below:

Wan-Qiong Huang prepared the Supplementary Information, performed cell culture and MTT assay of A549 and A549CIS cells, western blotting analysis, analysis of mitochondrial membrane potential and endoplasmic reticulum, DNA agarose gel electrophoresis assay, and detection of intracellular ROS.

Peng-Chao Ji synthesized and characterized the nitridoosmium(VI) complex $\mathrm{Na}\left[\mathrm{Os}{ }^{\mathrm{VI}}(\mathrm{N})(\operatorname{tpm})_{2}\right]$, and performed the cell culture of HeLa, HepG2, A2780 and MDA-MB-231cells, as well as the cytotoxicity test by means of MTT assay.

Fu-Ling Song, Chuan-Xian Wang and Tao Liu performed Human liver hepatocellular carcinoma tumor xenograft in nude mice, in vivo antitumor activity measurement.

Fu-Ling Song, Meiyang Li, Cuicui Yu performed cell culture of HepG2-stem and apoptosis analysis of HepG2-stem cells.

Hongzhi Guo: Proteomic data analysis.

Dr. Yong-Liang Huang collected X-ray diffraction data and resolved the structure of nitroosmium complex.

All authors reviewed the manuscript.

\section{Additional information Competing interests}

The author(s) declare no competing interests.

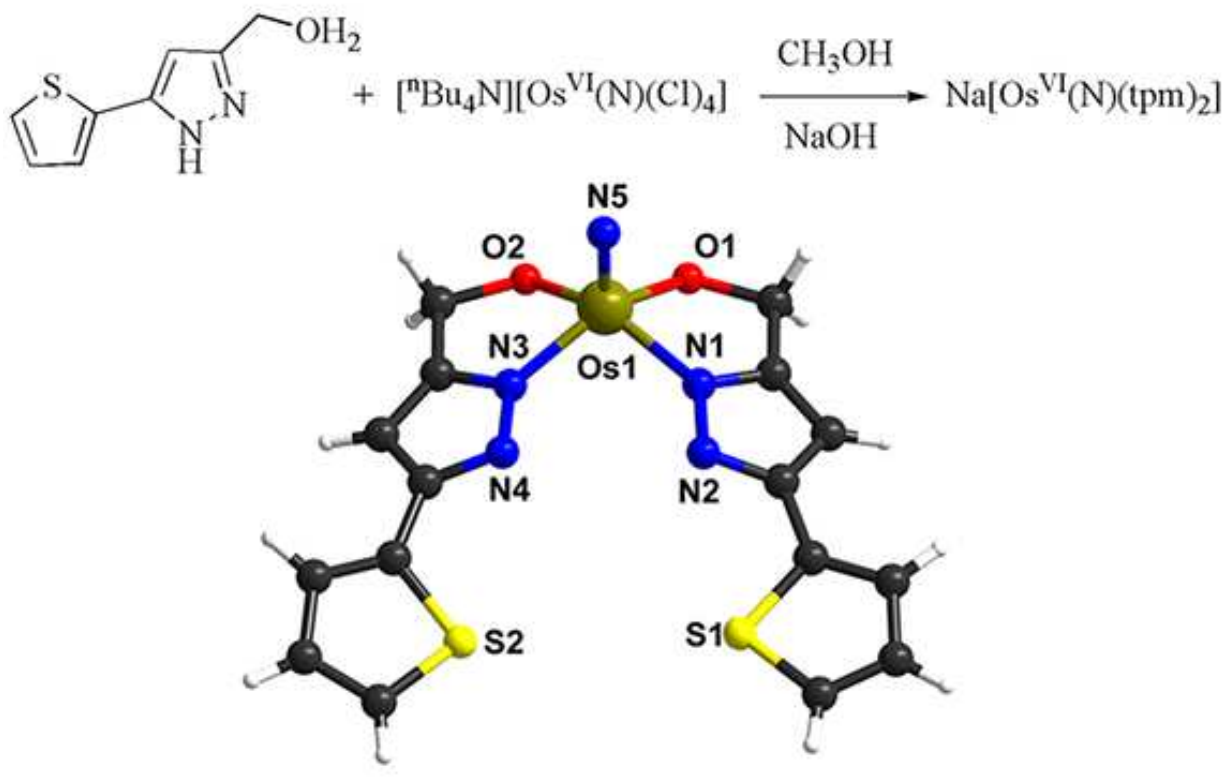

Figure 1. Synthesis and X-ray crystal structure of the Os complex. 
A

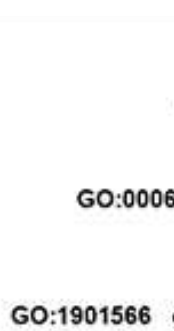

GO:1901566

organonitrogen compound biosynthetic process GO:0006986 response to unfolded protein GO:0016999 antibiotic metabolic process

GO:0065003 protein-containing complex assembly

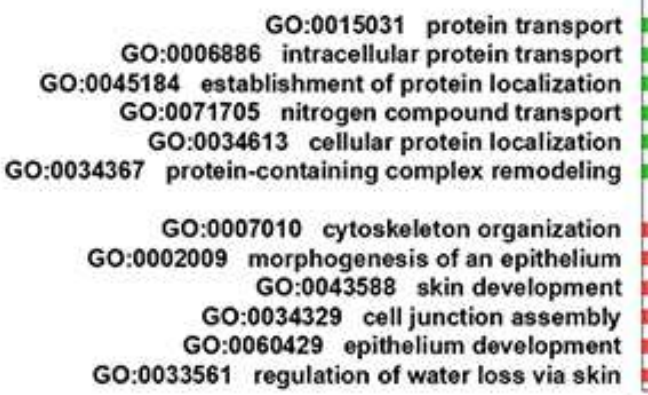

GO:0015031 protein transport O:0044772 mitotic cell cycle phase transition GO:0044843 cell cycle G1/S phase transition GO:0090304 nucleic acid metabolic process 4 cellular response to DNA damage stimulus
B

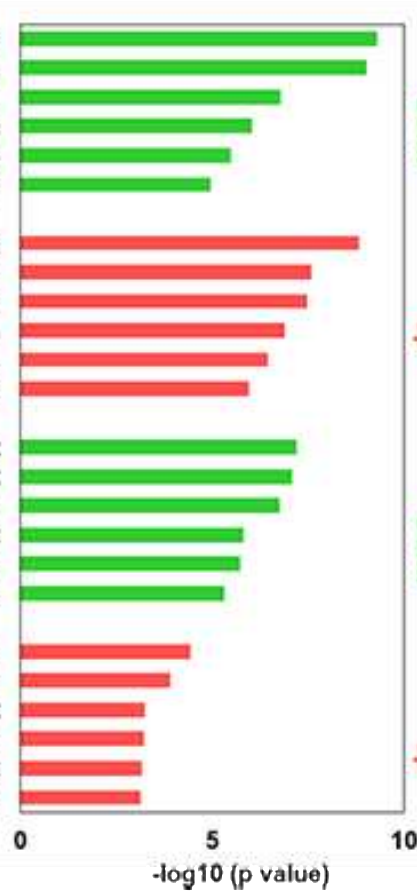

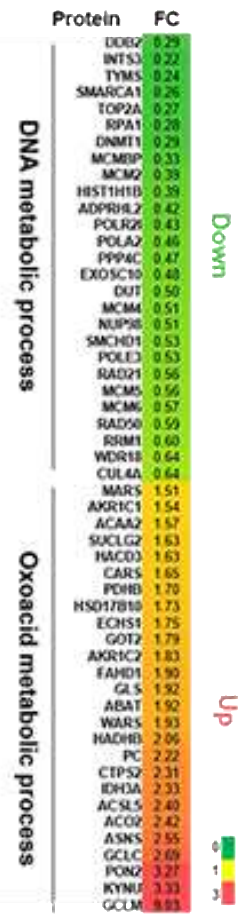

Figure 2. A) Gene ontology analysis for downregulated and upregulated proteins after 8 or $24 \mathrm{~h}$ of treatment with Os. B) The heat map shows the fold change of expression of the downregulated proteins in the terms of the DNA metabolic process and the upregulated proteins in terms of the oxoacid metabolic process after 24 hours Os treatment from panel A.

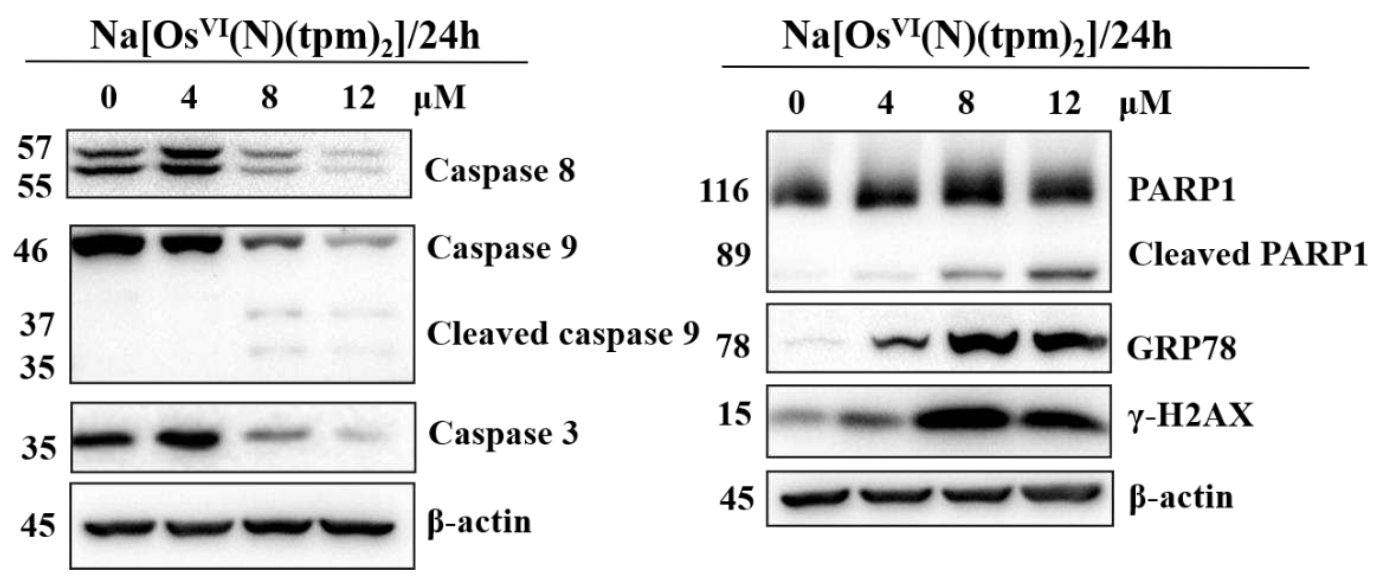

Figure 3. The expression levels of several proteins involved in apoptosis in HepG2 cells were assessed by Western blot after treatment with 4,8 or $12 \mu \mathrm{M} \mathrm{Na}\left[\mathrm{Os}^{\mathrm{VI}}(\mathrm{N})(\mathrm{tpm})_{2}\right]$ for $24 \mathrm{~h}$. Data are representative of three independent experiments. The fulllength blots are included in the Supplementary Information. (Supplementary Fig. S10) 


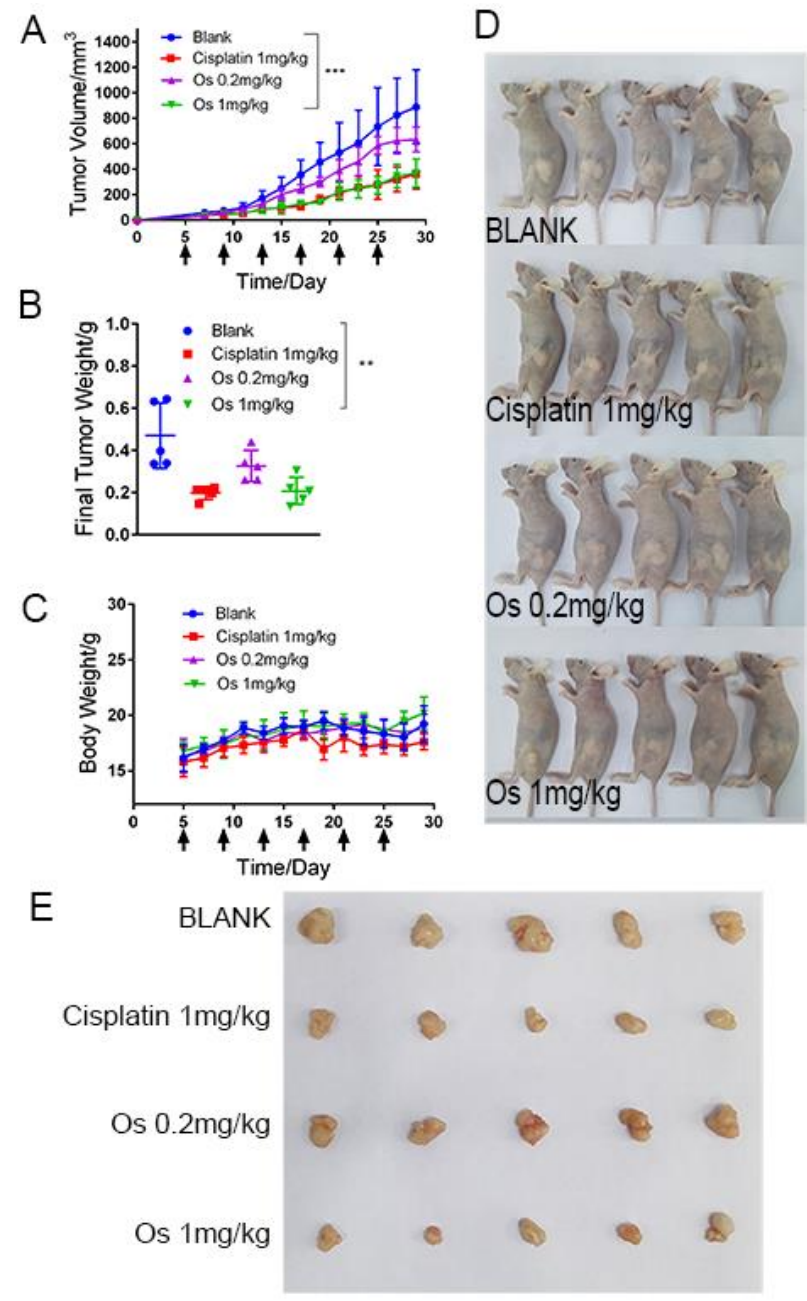

Figure 4. In vivo anticancer activity in nude mice bearing HepG2 tumour xenografts. A) Tumour growth curves. Tumour volume of the $1 \mathrm{mg} / \mathrm{kg}$ Os treated group vs vehicle control group, $\mathrm{P}<0.001$. B) Final tumour weights. Tumour weight of the $1 \mathrm{mg} / \mathrm{kg} \mathbf{O s}$ treated group vs vehicle control group, $\mathrm{P}<0.01$. C) Animal body weight growth curves. D) Photograph of the mice in all groups on day 29. E) Photograph of the tumours in all groups on day 29. Os represents $\mathrm{Na}\left[\mathrm{Os}^{\mathrm{VI}}(\mathrm{N})(\mathrm{tpm})_{2}\right]$. Data are presented as the mean \pm s.d. $(\mathrm{n}=5)$. 


\begin{tabular}{|l|l|l|}
\hline Cell lines & $\mathrm{Na}\left[\mathrm{Os}^{\mathrm{VI}}(\mathrm{N})(\mathrm{tpm})_{2}\right]$ & Cisplatin \\
\hline HeLa & $10.8 \pm 1.0$ & $13.4 \pm 0.7$ \\
\hline A2780 & $5.6 \pm 0.4$ & $15.9 \pm 0.9$ \\
\hline MDAMB231 & $9.1 \pm 0.6$ & $11.3 \pm 1.6$ \\
\hline HepG2 & $6.5 \pm 1.1$ & $4.9 \pm 0.3$ \\
\hline HepG2-stem & $8.2 \pm 0.8$ & $3.3 \pm 0.2$ \\
\hline A549 & $11.5 \pm 0.8$ & $12.3 \pm 1.5$ \\
\hline A549CIS & $15.3 \pm 2.0$ & $149.6 \pm 10.6$ \\
\hline LO-2 & $28.5 \pm 5.1$ & $5.0 \pm 0.3$ \\
\hline RI $^{\mathrm{a}}$ & 1.3 & 12.2 \\
\hline
\end{tabular}

[a] RF: resistance factor calculated as $\mathrm{RI}=\mathrm{IC}_{50}(\mathrm{~A} 549 \mathrm{CIS}) / \mathrm{IC}_{50}(\mathrm{~A} 549)$.

Table 1. Cytotoxic effects of the $\mathrm{Na}\left[\mathrm{Os}^{\mathrm{VI}}(\mathrm{N})(\mathrm{tpm})_{2}\right]$ complex and cisplatin on human cell lines. 


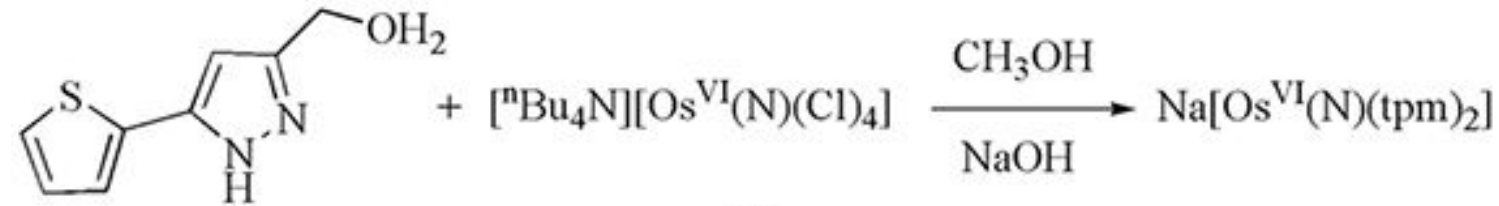

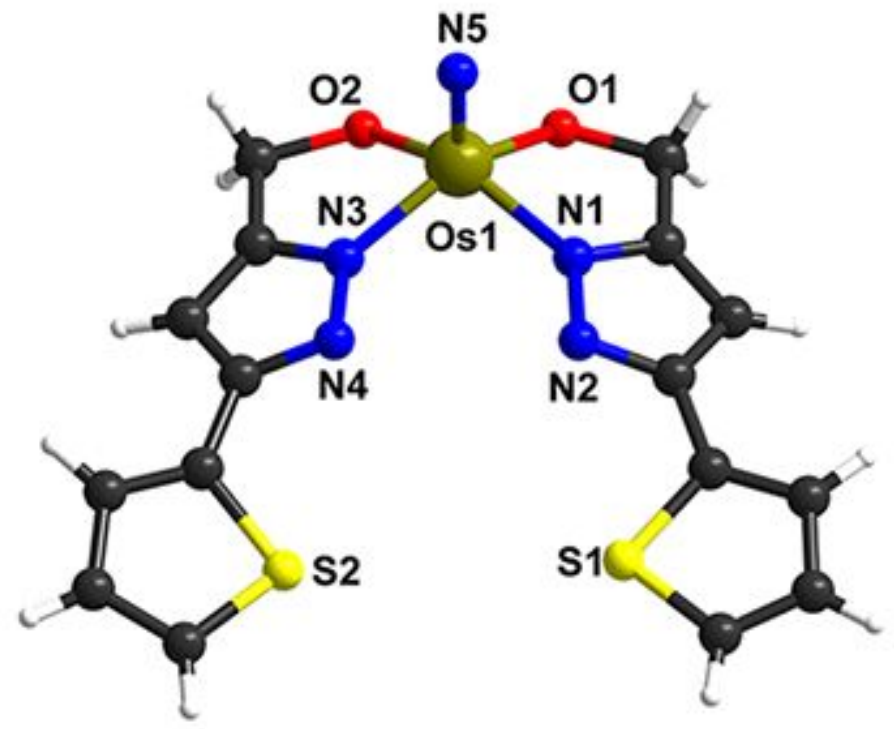

\section{Figure 1}

Synthesis and X-ray crystal structure of the Os complex.

A

GO Terms:
GO:0006259 DNA metabolic process
GO:0051276 chromosome organization
GO:0044772 mitotic cell cycle phase transition
GO:0044843 cell cycle G1/S phase transition
GO:0090304 nucleic acid metabolic process
GO:0006974 cellular response to DNA damage stimulus
GO:0043436 oxoacid metabolic process
GO:0043603 cellular amide metabolic process
organonitrogen compound biosynthetic process
GO:0006986 response to unfolded protein
GO:0016999 antibiotic metabolic process
protein-containing complex assembly
GO:0065003:0015031 protein transport
GO:0006886 intracellular protein transport
GO:0071705 nitrogen compound transport
GO:0034613 cellular protein localization
protein-containing complex remodeling
GO:0007010 cytoskeleton organization
GO:0045184
GO:0002009 morphogenesis of an epithelium
GO:0043588 skin development
GO:0034329 cell junction assembly
GO:0060429 epithelium development
GO:0033561 regulation of water loss via skin


Figure 2

A) Gene ontology analysis for downregulated and upregulated proteins after 8 or $24 \mathrm{~h}$ of treatment with Os. B) The heat map shows the fold change of expression of the downregulated proteins in the terms of the DNA metabolic process and the upregulated proteins in terms of the oxoacid metabolic process after 24 hours Os treatment from panel A.

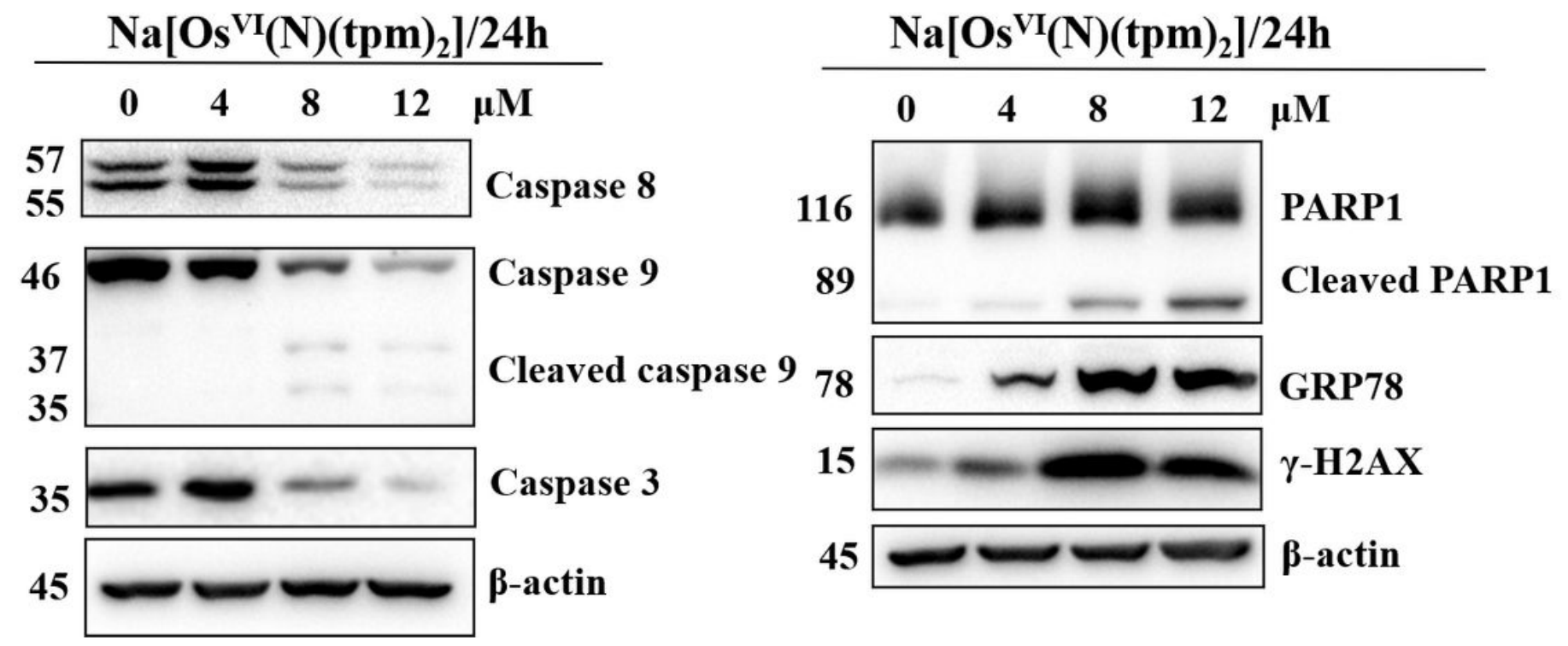

Figure 3

The expression levels of several proteins involved in apoptosis in HepG2 cells were assessed by Western blot after treatment with 4, 8 or $12 \mu \mathrm{M} \mathrm{Na}$ [OsVI(N)(tpm)2] for $24 \mathrm{~h}$. Data are representative of three independent experiments. The full-length blots are included in the Supplementary Information. (Supplementary Fig. S10) 


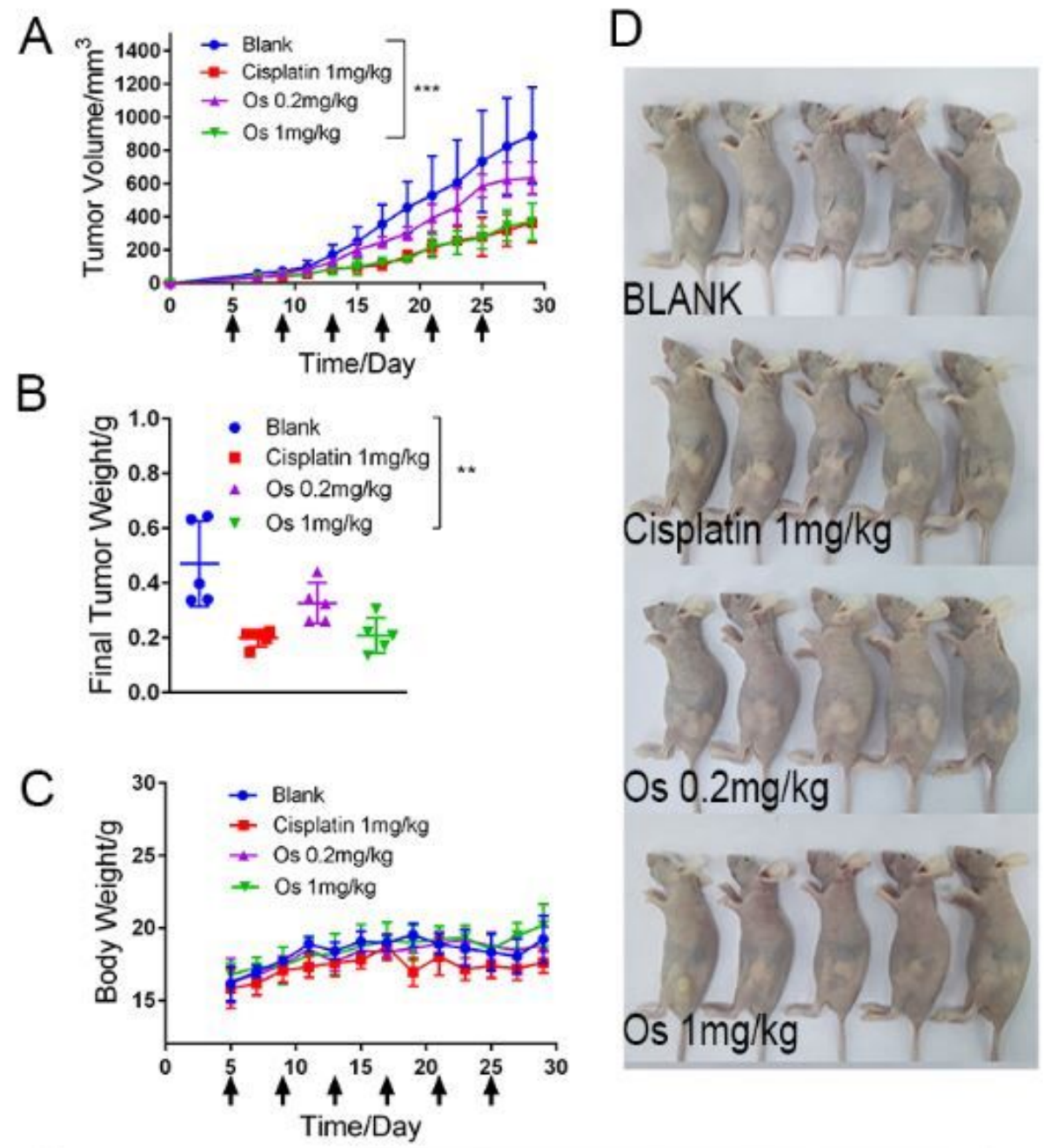

E BLANK

Cisplatin $1 \mathrm{mg} / \mathrm{kg}$

Os $0.2 \mathrm{mg} / \mathrm{kg}$

Os $1 \mathrm{mg} / \mathrm{kg}$
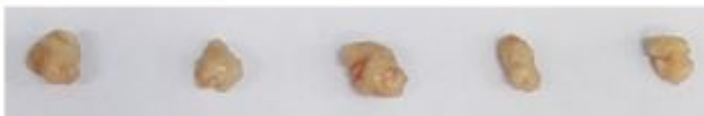

\author{
Os $0.2 \mathrm{mg} / \mathrm{kg}$
}
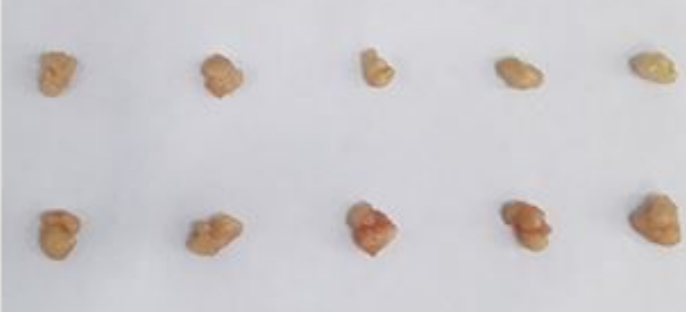
tumours in all groups on day 29. Os represents $\mathrm{Na}[\mathrm{Os} \varangle(\mathrm{N})(\mathrm{tpm}) 2]$. Data are presented as the mean $\pm \mathrm{s} . \mathrm{d}$. $(n=5)$.

\section{Supplementary Files}

This is a list of supplementary files associated with this preprint. Click to download.

- Supplementarylnformation.pdf 